\title{
SUSY Higgs boson flavor-changing neutral currents at the LHC
}

\author{
Santi Béjar ${ }^{\mathrm{ab}}, \underline{\text { Jaume Guasch }}^{\mathrm{c}}$, Joan Solà ${ }^{\mathrm{cb} *}$ \\ ${ }^{a}$ Grup de Física Teòrica, Universitat Autònoma de Barcelona, E-08193, Bellaterra, Barcelona, \\ Catalonia, Spain \\ bInstitut de Física d'Altes Energies, Universitat Autònoma de Barcelona, E-08193, Bellaterra, \\ Barcelona, Catalonia, Spain \\ 'HEP Group, Departament d'Estructura i Constituents de la Matèria, Universitat de Barcelona, \\ Diagonal 647, E-08028 Barcelona, Catalonia, Spain
}

UAB-FT-597, UB-ECM-PF-06/03, hep-ph/0601191

We compute and analyze the Flavor-Changing Neutral Current (FCNC) interactions of Minimal Supersymmetric Standard Model Higgs bosons $\left(h \equiv h^{0}, H^{0}, A^{0}\right)$ with heavy quarks (top and bottom), focusing on the strongly-interacting sector. We correlate the Higgs bosons production cross-section at the LHC with the FCNC decay branching ratios and find the maximum allowed values of the production rates, $\sigma(p p \rightarrow h \rightarrow$ $\left.q q^{\prime}\right) \equiv \sigma(p p \rightarrow h) \times B\left(h \rightarrow q q^{\prime}\right)\left(q q^{\prime} \equiv t c\right.$ or $\left.b s\right)$ after taking into account limits from low energy data on flavor-changing interactions. We single out the top quark channel, with a maximum production rate of $\sigma^{\max }(p p \rightarrow h \rightarrow t c) \simeq 10^{-3}-10^{-2} \mathrm{pb}$, as the most promising FCNC channel to be detected at the LHC.

\section{Introduction}

The possibility that Nature hides the last possible symmetry of the $S$-matrix, Supersymmetry (SUSY), in the form of a broken symmetry of the fundamental interactions is one of the most intriguing puzzles over the last 30 years of high energy physics. SUSY offers a most tantalizing paradigm to unify Particle Physics interactions with Gravity, and it might even provide a clue to a more fundamental superstring unification of all interactions. As such SUSY will be scrutinized in great detail at the LHC. It also offers a possible solution to the longstanding naturalness problem in the Higgs sector of the SM [1]. If SUSY is realized around the $\mathrm{TeV}$ scale, the LHC experiments shall be able to directly produce the SUSY particles for masses smaller than a few $\mathrm{TeV}$ [2]. Among the many strategies devised to hunt for SUSY, Flavor-Changing Neutral Currents (FCNC) offer perhaps a unique laboratory to seek for the new signs of physics beyond

${ }^{*}$ Contribution to the proceedings of 7 th International Symposium on Radiative Corrections, Shonan Village, Japan, October 2-7, 2005 the SM. In the SM the FCNC effects are completely absent at the tree-level. At one loop, however, they are possible, but then the contributions from the new particles enter on equal footing with those from SM particles. In certain regions of the parameter space the new physical effects may well dominate the SM contributions. This is particularly so when the SM one-loop effects turn out to be highly suppressed. In such situations the sole observation of these FCNC processes would be instant evidence of new physics. An example of this kind of appealing scenarios occurs within the FCNC physics of the SM Higgs boson $\left(H_{\mathrm{SM}}\right)$ interactions with quarks. For example, the FCNC vertex $H_{\mathrm{SM}} t c$ may lead (at one loop) to such rare decays as $t \rightarrow H_{\mathrm{SM}} c$ or $H_{\mathrm{SM}} \rightarrow t c$, depending on the mass of $H_{\mathrm{SM}}$. Both of these modes are extremely suppressed at one loop, with branching ratios of order $10^{-14}$ or less [3, 4], hence 10 orders of magnitude below other more conventional (and relatively well measured) FCNC processes like $b \rightarrow s \gamma$ [5].

The Minimal Supersymmetric Standard Model (MSSM) introduces new sources of FCNC interactions mediated by the strongly-interacting sec- 
tor $^{1}$. They are produced by the misalignment of the quark mass matrix with the squark mass matrix, and the main parameter characterizing these interactions is the non-flavor-diagonal term in the squark-mass-matrix, which we parameterize in the standard fashion [8] as $\left(M^{2}\right)_{i j}=$ $\delta_{i j} \tilde{m}_{i} \tilde{m}_{j}(i \neq j), \tilde{m}_{i}$ being the flavor-diagonal mass-term of the $i$-flavor squark. Since there are squarks of different chiralities, there are different $\delta_{i j}$ parameters for the different chirality mixings. In this work we will assume flavor-mixing only among the Left-Chiral squarks, since these mixing terms are expected to be the largest ones by Renormalization Group analysis [9].

Some work in relation with the MSSM Higgs bosons FCNCs has already been performed $[4,7$, $10,11]$. In this work we compute and analyze the production of any MSSM Higgs boson $(h=$ $h^{0}, H^{0}, A^{0}$ ) at the LHC, followed by the one-loop FCNC decay $h \rightarrow b s$ or $h \rightarrow t c$, and we find the maximum production rates of the combined cross-section:

$$
\begin{aligned}
\sigma\left(p p \rightarrow h \rightarrow q q^{\prime}\right) & \equiv \sigma(p p \rightarrow h X) B\left(h \rightarrow q q^{\prime}\right) \\
B\left(h \rightarrow q q^{\prime}\right) & \equiv \frac{\Gamma\left(h \rightarrow q \bar{q}^{\prime}+\bar{q} q^{\prime}\right)}{\sum_{i} \Gamma\left(h \rightarrow X_{i}\right)}
\end{aligned}
$$

$q q^{\prime}$ being a pair of heavy quarks $\left(q q^{\prime} \equiv b s\right.$ or $t c)$, taking into account the restrictions from the experimental determination of $B(b \rightarrow s \gamma)$ [5]. For other signals of SUSY FCNC at the LHC, without Higgs bosons couplings see Ref. [6].

\section{Computation setup}

In this section we give a summarized explanation of the computation. For further details see Refs. $[4,11]$.

We include the full one-loop SUSY-QCD contributions to the FCNC partial decay widths $\Gamma\left(h \rightarrow q q^{\prime}\right)$ in the observable (11).

The Higgs sector parameters (masses and CPeven mixing angle $\alpha$ ) have been treated using the leading $m_{t}$ and $m_{b} \tan \beta$ approximation to the one-loop result [12].

The Higgs bosons total decay widths $\Gamma(h \rightarrow X)$ are computed at leading order, including all the

${ }^{1}$ For a brief description of these interactions see e.g. Ref. [6], for details see [7]. relevant channels: $\Gamma\left(h \rightarrow f \bar{f}, Z Z, W^{+} W^{-}, g g\right)$. The off-shell decays $\Gamma\left(h \rightarrow Z Z^{*}, W^{ \pm} W^{\mp *}\right)$ have also been included. This is necessary to consistently compute the total decay width of $\Gamma\left(h^{0} \rightarrow\right.$ $X)$ in regions of the parameter space where the maximization of the cross-section (1I) is obtained at the expense of greatly diminishing the partial decay widths of the two-body process $h^{0} \rightarrow b \bar{b}$ (due to dramatic quantum effects that may reduce the CP-even mixing angle $\alpha$ to small values [13]). The one-loop decay rate $\Gamma(h \rightarrow g g)$ has been taken from [14] and the off-shell decay partial widths have been recomputed explicitly.

The MSSM Higgs boson production crosssections have been computed using the programs HIGLU 2.101 and PPHTT $1.1 \quad[14,15]$. These programs include the following channels: gluongluon fusion, and associated production with topand bottom-quarks. We have used the leading order approximation for all channels. The QCD renormalization scale is set to the default value for each program. We have used the set of CTEQ4L PDF [16].

For the constraints on the FCNC parameters, we use $B(b \rightarrow s \gamma)=(2.1-4.5) \times 10^{-4}$ as the experimentally allowed range within three standard deviations [5]. We also require that the sign of the $b \rightarrow s \gamma$ amplitude is the same as in the SM $[17]^{2}$.

Running quark masses $\left(m_{q}(Q)\right)$ and strong coupling constants $\left(\alpha_{s}(Q)\right)$ are used throughout, with the renormalization scale set to the decaying Higgs boson mass in the decay processes.

Given this setup, we have performed a MonteCarlo maximization [18] of the cross-section (11) over the MSSM parameter space, keeping the parameter $\tan \beta$ fixed, and under the simplification that the squark and gluino soft-SUSY-breaking parameter masses are at the same scale:

$m_{\tilde{d}_{L}}=m_{\tilde{d}_{R}}=m_{\tilde{u}_{R}}=m_{\tilde{g}} \equiv M_{\mathrm{SUSY}} \quad$.

Although we have performed a full one-loop computation, involving the exact diagonalization of the $6 \times 6$ squark mass matrix, it is enlightening to look at the approximate leading expressions to

${ }^{2}$ This constraint automatically excludes the fine-tuned regions of Ref. [4]. 


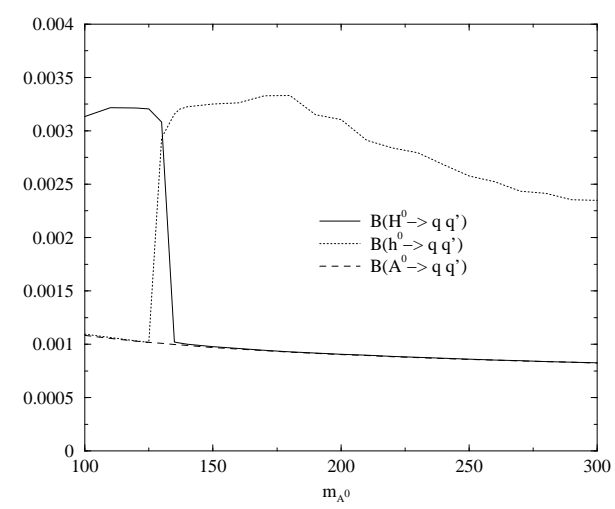

Figure 1. The maximum value of $B(h \rightarrow b s)$ as a function of $m_{A^{0}}$, for $\tan \beta=50$.

understand the qualitative trend of the results. The SUSY-QCD contribution to the $b \rightarrow s \gamma$ amplitude can be approximated to:

$$
A^{S Q C D}(b \rightarrow s \gamma) \sim \delta_{23} \frac{m_{b}\left(\mu-A_{b} \tan \beta\right)}{M_{\mathrm{SUSY}}^{2}},
$$

whereas the MSSM Higgs bosons FCNC effective couplings behave as:

$g_{h q \bar{q}^{\prime}} \sim \delta_{23} \frac{-\mu m_{\tilde{g}}}{M_{S U S Y}^{2}}\left\{\begin{array}{cc}\sin \left(\beta-\alpha_{\mathrm{eff}}\right) & \left(H^{0}\right) \\ \cos \left(\beta-\alpha_{\mathrm{eff}}\right) & \left(h^{0}\right) \\ 1 & \left(A^{0}\right)\end{array}\right.$.

The different structure of the amplitudes in eqs. (3) and (4), will permit to obtain and appreciable FCNC Higgs boson decay rate, while the prediction for $B(b \rightarrow s \gamma)$ stays inside the experimentally allowed range.

\section{Bottom-strange channel}

For the analysis of the bottom-strange production channel, we study first the Higgs boson branching ratio (11). Figure 11 shows the maximum value of $B(h \rightarrow b s)$ as a function of the pseudoscalar Higgs boson mass $m_{A^{0}}$. We observe that fairly large values of $B\left(h^{0} \rightarrow b s\right) \sim 0.3 \%$ are obtained. Table 1 shows the actual values of the maximum branching ratios, and the parameters that provide them for each Higgs boson. Let us discuss first the general trend, which is valid for all studied processes: the maximum is attained at large $M_{\text {SUSY }}$ and moderate $\delta_{23}$. The SUSY-QCD contribution to $b \rightarrow s \gamma(3)$ decreases with $M_{\mathrm{SUSY}}$, therefore to keep $B(b \rightarrow s \gamma)$ in the allowed range when $M_{\text {SUSY }}$ is small, it has to be compensated with a low value of $\delta_{23}$, providing a small FCNC effective coupling (4). On the other hand, at large $M_{\text {SUSY }}$ the second factor in eq. (3) decreases, allowing a larger value of $\delta_{23}$. Thus, the first factor in eq. (4) grows, but the second factor in eq. (4) stays fixed (provided that $|\mu| \sim M_{\mathrm{SUSY}}$ ), overall providing a larger value of the effective coupling. On the other hand, a too large value of $\delta_{23}$ has to be compensated by a small value of $|\mu| / M_{\mathrm{SUSY}}$ in (3), provoking a reduction in (4). In the end, the balance of the various interactions involved produces the results of Table 1

The maximum value of the branching ratio for the lightest Higgs boson channel is obtained in the small $\alpha_{\text {eff }}$ scenario [13]. In this scenario the coupling of bottom quarks to $h^{0}$ is extremely suppressed. The large value of $B\left(h^{0} \rightarrow b s\right)$ is obtained because the total decay width $\Gamma\left(h^{0} \rightarrow X\right)$ in the denominator of (1) tends to zero (Table 1), and not because a large FCNC partial decay width in its numerator [4].

The leading production channel of $h^{0}$ at the LHC at high $\tan \beta$ is the associated production with bottom quarks, and therefore the $h^{0}$ production will be suppressed when $B\left(h^{0} \rightarrow b s\right)$ is enhanced. We have to perform a combined analysis of the full process (11) to obtain the maximum production rate of FCNC Higgs bosons meditated 


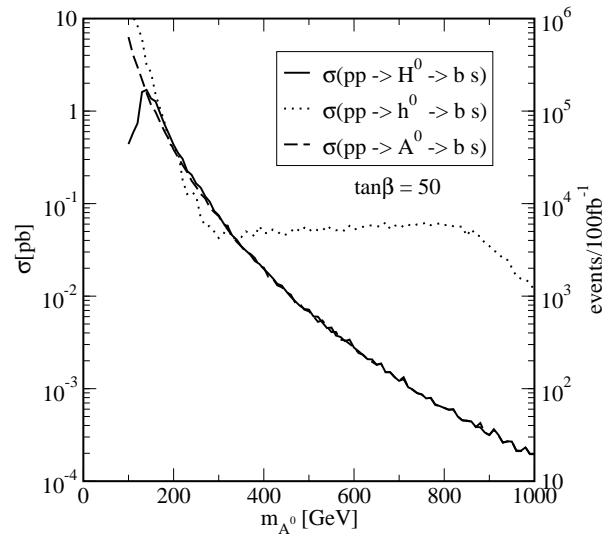

Figure 2. Maximum SUSY-QCD contributions to $\sigma(p p \rightarrow h \rightarrow b s)$ as a function of $m_{A^{0}}, \tan \beta=50$.

events at the LHC. Figure 2 and Table 2 show the result of the maximization of the production cross-section (11). The central column of Table 2 shows that when performing the combined maximization $\Gamma\left(h^{0} \rightarrow X\right)$ has a much larger value, and therefore the maximum of the combined crosssection is not obtained in the small $\alpha_{\text {eff }}$ scenario. The number of expected events at the LHC is around 50,000 events $/ 100 \mathrm{fb}^{-1}$. While it is a large number, the huge $b$-quark background at the LHC will most likely prevent its detection. Note, however, that the maximum FCNC branching ratios are around $10^{-4}-10^{-3}$, which is at the same level that the already measured $B(b \rightarrow s \gamma)$.

\section{Top-charm channel}

The results of the numerical scan for this channel are similar to the $b s$ channel, so we will focus mainly on the differences. Figure 3 shows the maximum value of the production cross-section $\sigma(p p \rightarrow h \rightarrow t c)$ as a function of $m_{A^{0}}$, while Table 3 shows the actual value of the maximum, together with the SUSY parameters that provide them, for $m_{A^{0}}=300 \mathrm{GeV}$. Only the heavy neutral Higgs bosons contribute to this channel. The general trend explained in section 3 is also valid here $^{3}$. From Table 3 we would expect some 300 events $/ 100 \mathrm{fb}^{-1}$ at the LHC. However, the maximum is attained at low $\tan \beta$, and we have fixed a moderate value of $\tan \beta=5$. By taking lower values of $\tan \beta$ we find that the number of events grows fast [11], e.g. up to $\sim(500,900,2000)$ if we would have chosen $\tan \beta=(4,3,2)$ respectively. Due to the single top quark signature the detection of this channel should be feasible at the LHC.

\section{Conclusions}

We have performed a computation of Higgs boson mediated FCNC events at the LHC. Alternative effects of SUSY FCNC have been considered in Ref. [6]. Note from eq. (4) that the present effects, though smaller than the latter, do not decouple with $M_{\mathrm{SUSY}}=m_{\tilde{g}}=|\mu|$. The maximum expected branching ratio for the $b s$ channel is large, and we expect a maximum of $\sim 50,000$ events $/ 100 \mathrm{fb}^{-1}$ in this channel at the LHC, which might be difficult to detect due to the huge background. For the $t c$ channel we expect a maximum of $\sigma^{\max }(p p \rightarrow h \rightarrow t c) \simeq 10^{-3}-10^{-2} \mathrm{pb}$, which means several thousand events per $100 \mathrm{fb}^{-1}$ at the

\footnotetext{
${ }^{3}$ Recall that the $\delta_{i j}$ parameters in the up-sector are related to the corresponding parameters in the down-sector by the Cabibbo-Kobayashi-Maskawa matrix, see e.g. [8], and are therefore constrained by $B(b \rightarrow s \gamma)$.
} 


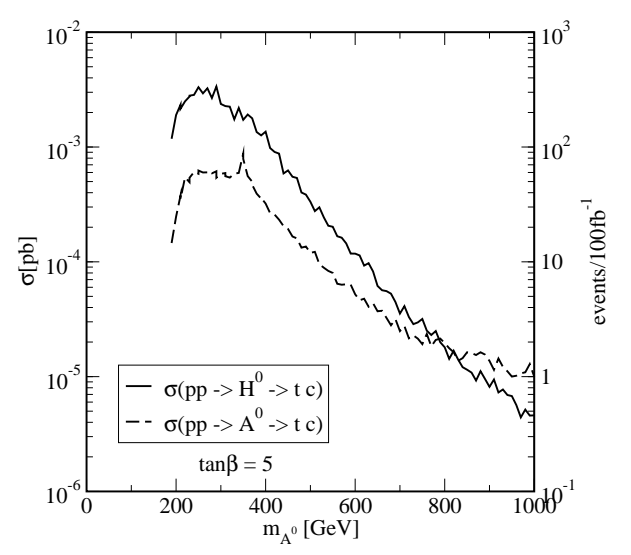

Figure 3. Maximum SUSY-QCD contributions to $\sigma(p p \rightarrow h \rightarrow t c)$ as a function of $m_{A^{0}}, \tan \beta=5$.

LHC. Due to the single top quark signature they should be easier to detect than the $b s$ channel, providing the key to a new door to study physics beyond the Standard Model. It is now an experimental challenge to use this key to open the door, and prove that these events can be effectively be separated from the background.

Acknowledgements The work of SB has been supported by CICYT (FPA2002-00648), by the EU (HPRN-CT-2000-00152), and by DURSI (2001SGR-00188); JG by a Ramon y Cajal contract from MEC (Spain); JG and JS in part by MEC and FEDER (2004-04582-C02-01) and by DURSI (2005SGR00564).

\section{REFERENCES}

1. H. Haber, G. Kane, Phys.Rept.117, 75 (1985).

2. G. Weiglein et al., hep-ph/0410364 G. Degrassi et al., Acta Phys.Polon.B35, 2711 (2004).

3. B. Mele, S. Petrarca, A. Soddu, Phys. Lett. B435, 401 (1998), hep-ph/9805498 S. Béjar, J. Guasch, J. Solà, Nucl. Phys. B600, 21 (2001), hep-ph/0011091 ibid. B675, 270 (2003), hep-ph/0307144

4. S. Béjar, F. Dilmé, J. Guasch, J. Solà, JHEP 08, 018 (2004), hep-ph/0402188

\begin{tabular}{|c||c|c|}
\hline$h$ & $H^{0}$ & $A^{0}$ \\
\hline \hline$\sigma(p p \rightarrow h \rightarrow t c)$ & $2.4 \times 10^{-3} \mathrm{pb}$ & $5.8 \times 10^{-4} \mathrm{pb}$ \\
\hline events $/ 100 \mathrm{fb}^{-1}$ & 240 & 58 \\
\hline$B(h \rightarrow t c)$ & $1.9 \times 10^{-3}$ & $5.7 \times 10^{-4}$ \\
\hline$\Gamma(h \rightarrow X)$ & $0.41 \mathrm{GeV}$ & $0.39 \mathrm{GeV}$ \\
\hline$\delta_{23}$ & $10^{-0.10}$ & $10^{-0.13}$ \\
\hline$m_{\tilde{q}}$ & $880 \mathrm{GeV}$ & $850 \mathrm{GeV}$ \\
\hline$A_{t}$ & $-2590 \mathrm{GeV}$ & $2410 \mathrm{GeV}$ \\
\hline$\mu$ & $-700 \mathrm{GeV}$ & $-930 \mathrm{GeV}$ \\
\hline$B(b \rightarrow s \gamma)$ & $4.13 \times 10^{-4}$ & $4.47 \times 10^{-4}$ \\
\hline
\end{tabular}

Table 3

As in Table 2 but for $\sigma(p p \rightarrow h \rightarrow t c), m_{A^{0}}=$ $300 \mathrm{GeV}$ and $\tan \beta=5$.

5. S. Eidelman et al., Phys.Lett.B592, 1 (2004).

6. J. Guasch, W. Hollik, S. Peñaranda, J. Solà, these proceedings, hep-ph/0601218

7. J. Guasch, J. Solà, Nucl. Phys. B562, 3 (1999), hep-ph/9906268

8. F. Gabbiani et al., Nucl. Phys. B477, 321 (1996), hep-ph/9604387 M. Misiak, S. Pokorski, J. Rosiek, Adv. Ser. Direct. High Energy Phys. 15 (1998), eds. A.J. Buras, M. Lindner, hep-ph/9703442

9. M. J. Duncan, Nucl. Phys. B221, 285 (1983).

10. J. Guasch, proc. of QEMSSM, 256, ed. J. Solà, hep-ph/9710267 J. Guasch, J. Solà, proc. of LCWS 99, 196, eds. E. Fernández, A. Pacheco, hep-ph/9909503 S. Béjar, J. Guasch, J. Solà, proc. of RADCOR 2000, hep-ph/0101294 A. M. Curiel, M. J. Herrero, D. Temes, Phys. Rev. D67, 075008 (2003), hep-ph/0210335 D. A. Demir, Phys. Lett. B571, 193 (2003), hep-ph/0303249 A. M. Curiel et al., Phys. Rev. D69, 075009 (2004), hep-ph/0312135 S. Heinemeyer et al., Eur. Phys. J. C37, 481 (2004), hep-ph/0403228 J. A. Aguilar-Saavedra, Acta Phys. Polon. B35, 2695 (2004), hep-ph/0409342 T. Hahn et al., hep-ph/0512315

11. S. Béjar, J. Guasch, J. Solà, JHEP 10, 113 (2005), hep-ph/0508043

12. A. Yamada, Z. Phys. C61, 247 (1994); P. Chankowski, S. Pokorski, J. Rosiek, Nucl. Phys. B423, 437 (1994), hep-ph/9303309 A. Dabelstein, Z. Phys. C67, 495 (1995), 
hep-ph/9409375 Nucl. Phys. B456, 25 (1995), hep-ph/9503443

13. M. Carena, S. Mrenna, C. Wagner, Phys. Rev. D62, 055008 (2000), hep-ph/9907422

M. Carena et al., Eur. Phys. J. C26, 601 (2003), hep-ph/0202167

14. M. Spira et al., Nucl. Phys. B453, 17 (1995), hep-ph/9504378.

15. M. Spira, hep-ph/9510347 Fortsch. Phys. 46, 203 (1998), hep-ph/9705337

16. H. L. Lai et al., Phys. Rev. D55, 1280 (1997), hep-ph/9606399.

17. P. Gambino, U. Haisch, M. Misiak, Phys. Rev. Lett. 94, 061803 (2005), hep-ph/0410155

18. O. Brein, Comput. Phys. Commun. 170, 42 (2005), hep-ph/0407340 